\title{
Newton Interpolation in Fejér and Chebyshev Points
}

\author{
By Bernd Fischer* and Lothar Reichel**
}

\begin{abstract}
Let $\Gamma$ be a Jordan curve in the complex plane, and let $\Omega$ be the compact set bounded by $\Gamma$. Let $f$ denote a function analytic on $\Omega$. We consider the approximation of $f$ on $\Omega$ by a polynomial $p$ of degree less than $n$ that interpolates $f$ in $n$ points on $\Gamma$. A convenient way to compute such a polynomial is provided by the Newton interpolation formula. This formula allows the addition of one interpolation point at a time until an interpolation polynomial $p$ is obtained which approximates $f$ sufficiently accurately. We choose the sets of interpolation points to be subsets of sets of Fejer points. The interpolation points are ordered using van der Corput's sequence, which ensures that $p$ converges uniformly and maximally to $f$ on $\Omega$ as $n$ increases. We show that $p$ is fairly insensitive to perturbations of $f$ if $\Gamma$ is smooth and is scaled to have capacity one. If $\Gamma$ is an interval, then the Fejer points become Chebyshev points. This special case is also considered. A further application of the interpolation scheme is the computation of an analytic continuation of $f$ in the exterior of $\Gamma$.
\end{abstract}

1. Introduction. Let $\Omega$ be a compact set in the complex plane $\mathbb{C}$, and assume that the boundary $\Gamma$ of $\Omega$ is a Jordan curve. Let $\varphi$ be the analytic function which maps $\{w:|w|>1\}$ conformally onto $\Omega_{c}:=\mathbb{C} \backslash \Omega$ so that $\varphi(\infty)=\infty$ and $\varphi^{\prime}(\infty)>0$. We assume that $\varphi$ is defined so as to be continuous and univalent for $1 \leq|w|<\infty$. The mapping $\varphi$ then has the Laurent expansion

$$
\varphi(w)=c w+d_{0}+d_{1} w^{-1}+d_{2} w^{-2}+\cdots, \quad|w|>1,
$$

where $c$ is the capacity of $\Omega$, see Gaier [5], Walsh [12]. For future reference we note that the capacity depends on the scaling of $\Omega$. Any set of $n$ points $\left\{z_{k, n}\right\}_{k=0}^{n-1} \subset \Gamma$ such that for some constant $\alpha \in \mathbf{R}$, and $i:=\sqrt{-1}$,

$$
z_{k, n}=\varphi\left(\exp \left(2 \pi i \frac{k}{n}+i \alpha\right)\right), \quad 0 \leq k<n,
$$

is called a set of Fejér points [5].

Example 1.1. Let $\Omega$ be the ellipse $E(a, b):=\left\{z=x+i y:(x / a)^{2}+(y / b)^{2} \leq 1\right\}$ for some positive constants $a, b$. Then

$$
\varphi(w)=\frac{1}{2}(a+b) w+\frac{1}{2}(a-b) w^{-1}
$$

and the capacity of $\Omega$ is $c=\frac{1}{2}(a+b)$. In particular, equidistant points on a circle are Fejér points.

Let $f$ be a function analytic on $\Omega$, and introduce the family of curves in $\Omega_{c}$

$$
\Gamma_{\rho}:=\{\varphi(w):|w|=\rho\}, \quad \rho>1 .
$$

Received May 9, 1988.

1980 Mathematics Subject Classification (1985 Revision). Primary 65D05, 65E05, $30 \mathrm{E} 05$.

Key words and phrases. Polynomial interpolation, Newton form, Fejér points, Chebyshev points, van der Corput's sequence, complex approximation, analytic continuation.

* Research supported by Bergen Scientific Centre.

** Research supported in part by the National Science Foundation under Grant DMS-870416. On leave from Department of Mathematics, University of Kentucky, Lexington, KY 40506. 
Then there is a largest constant $\rho(f)>1$ such that $f$ is analytic in the interior of $\Gamma_{\rho(f)}$. Let $p_{n-1}$ denote the polynomial of degree less than $n$ that interpolates $f$ in some set of $n$ Fejér points. It is well known, see [5], [12], that

$$
\varlimsup_{n \rightarrow \infty}\left\|f-p_{n-1}\right\|_{\Gamma}^{1 / n}=1 / \rho(f)
$$

where for $g \in C(\Gamma)$ we have

$$
\|g\|_{\Gamma}:=\max _{z \in \Gamma}|g(z)| .
$$

Let $p_{n-1}^{*}$ be the polynomial of degree less than $n$ of best approximation to $f$ on $\Omega$, i.e.,

$$
\left\|f-p_{n-1}^{*}\right\|_{\Gamma} \leq\left\|f-\tilde{p}_{n-1}\right\|_{\Gamma}
$$

for all polynomials $\tilde{p}_{n-1}$ of degree less than $n$. Then, see [5], [12],

$$
\varlimsup_{n \rightarrow \infty}\left\|f-p_{n-1}^{*}\right\|_{\Gamma}^{1 / n}=1 / \rho(f) .
$$

Formulas (1.4) and (1.6) express in the terminology of Walsh [12, Chapter 4] that $p_{n-1}$ converges maximally to $f$ as $n$ increases, i.e., the geometric rate of convergence is the best possible. In view of this and the fact that $p_{n-1}$ is much simpler to compute than $p_{n-1}^{*}$, it is often attractive to compute $p_{n-1}$ instead of $p_{n-1}^{*}$ when a polynomial approximation of $f$ on $\Omega$ is desired.

A disadvantage with interpolation in sets of Fejér points is that a polynomial $p_{n}$ of degree $\leq n$ that interpolates $f$ in a set of $n+1$ Fejér points cannot generally be computed as a simple modification of $p_{n-1}$. The purpose of the present paper is to describe a selection of interpolation points $\xi_{j}$ so that the interpolation polynomial can be written in Newton form, i.e., we define the interpolation polynomial

$$
q_{n-1}(z):=\left[\xi_{0}\right] f+\sum_{j=1}^{n-1}\left(\left[\xi_{0}, \xi_{1}, \ldots, \xi_{j}\right] f\right) \prod_{k=0}^{j-1}\left(z-\xi_{k}\right)
$$

where $\left[\xi_{j}\right] f:=f\left(\xi_{j}\right), 0 \leq j \leq n$, and

$$
\left[\xi_{0}, \xi_{1}, \ldots, \xi_{j}\right] f:=\left(\left[\xi_{0}, \xi_{1}, \ldots, \xi_{j-1}\right] f-\left[\xi_{1}, \xi_{2}, \ldots, \xi_{j}\right] f\right) /\left(\xi_{0}-\xi_{j}\right)
$$

for $1 \leq j<n$.

From (1.7) we can in a simple manner quickly compute $q_{n}$ if $q_{n-1}$ is known. Moreover, our scheme avoids the computation of sets of Fejér points (1.2) for many consecutive values of $n$.

We define the interpolation points $\xi_{j}$ by using the van der Corput sequence $\left\{c_{k}\right\}_{k=0}^{\infty}$, see Hlawka [10, pp. 93-94]. Let the integer $k, 0 \leq k<\infty$, have the binary representation

$$
k=\sum_{j=0}^{\infty} k_{j} 2^{j}, \quad k_{j} \in\{0,1\} .
$$

Then the $c_{k} \in[0,1)$ are given by

$$
c_{k}:=\sum_{j=0}^{\infty} k_{j} 2^{-j-1}
$$


and the interpolation points $\xi_{k}$ are defined by

$$
\begin{gathered}
\theta_{k}:=2 \pi c_{k}, \\
\xi_{k}:=\varphi\left(\exp \left(i \theta_{k}\right)\right) .
\end{gathered}
$$

Example 1.2. The $c_{k}$ for $0 \leq k<2^{l}$ can be determined by bit reversal of $k$ with respect to $2^{l}$. Let $b(k, l)$ be the value of the integer obtained by bit reversal of $0 \leq k<2^{l}$ with respect to $2^{l}$, i.e.,

$$
b(k, l):=2^{l-1} \sum_{j=0}^{l-1} k_{j} 2^{-j} .
$$

Then $c_{k}=2^{-l} b(k, l)$.

\begin{tabular}{|c|c|c|c|c|c|}
\hline$k$ & $\begin{array}{c}\text { binary repr. } \\
\text { of } k\end{array}$ & $\begin{array}{c}\text { binary repr. of } k \\
\text { bit-reversed } \\
\text { w.r.t. } 8\end{array}$ & $b(k, 3)$ & $c_{k}$ & $\theta_{k}$ \\
\hline 0 & $\begin{array}{lll}0 & 0 & 0\end{array}$ & $\begin{array}{lll}0 & 0 & 0\end{array}$ & 0 & 0 & 0 \\
\hline 1 & $\begin{array}{lll}0 & 0 & 1\end{array}$ & $\begin{array}{lll}1 & 0 & 0\end{array}$ & 4 & $1 / 2$ & $\pi$ \\
\hline 2 & $\begin{array}{lll}0 & 1 & 0\end{array}$ & $\begin{array}{lll}0 & 1 & 0\end{array}$ & 2 & $1 / 4$ & $\pi / 2$ \\
\hline 3 & $\begin{array}{lll}0 & 1 & 1\end{array}$ & $\begin{array}{lll}1 & 1 & 0\end{array}$ & 6 & $3 / 4$ & $3 \pi / 2$ \\
\hline 4 & $\begin{array}{lll}1 & 0 & 0\end{array}$ & $\begin{array}{lll}0 & 0 & 1\end{array}$ & 1 & $1 / 8$ & $\pi / 4$ \\
\hline 5 & $\begin{array}{lll}1 & 0 & 1\end{array}$ & $\begin{array}{lll}1 & 0 & 1\end{array}$ & 5 & $5 / 8$ & $5 \pi / 4$ \\
\hline 6 & $\begin{array}{lll}1 & 1 & 0\end{array}$ & $\begin{array}{lll}0 & 1 & 1\end{array}$ & 3 & $3 / 8$ & $3 \pi / 4$ \\
\hline 7 & $\begin{array}{lll}1 & 1 & 1 \\
\end{array}$ & $\begin{array}{lll}1 & 1 & 1 \\
\end{array}$ & 7 & $7 / 8$ & $7 \pi / 4$ \\
\hline
\end{tabular}

TABLE 1.1

Bit reversal and the van der Corput sequence.

Example 1.3. For any integer $l \geq 0$ the set $\left\{\xi_{k}\right\}_{k=0}^{2^{l}-1}$ is a set of Fejér points. This follows from $\{b(k, l)\}_{k=0}^{2^{l}-1}=\{k\}_{k=0}^{2^{l}-1}$. Therefore, $\left\{\exp \left(i \theta_{k}\right)\right\}_{k=0}^{2^{l}-1}$ is a set of equidistant points on the unit circle. This is illustrated in Table 1.1 for $l=3$.

Example 1.3 suggests that the nodes $\xi_{k}$ can be thought of as being determined by a suitable enumeration of the points in some set of Fejér points. Properties of the $\xi_{j}$ are discussed in Section 2. There, we also consider the case $\Gamma=[-2,2]$, and the interpolation points

$$
\left\{\begin{array}{l}
x_{0}:=-2, \\
x_{k}:=2 \cos \left(\pi c_{k-1}\right), \quad k=1,2,3, \ldots,
\end{array}\right.
$$

where the choice of $[-2,2]$ rather than $[-1,1]$ is for stability reasons.

Example 1.4. For any integer $l \geq 2$, the set $\left\{x_{k}\right\}_{k=0}^{2^{l}}$ is the set of extreme points of the Chebyshev polynomial $T_{2^{l}}(x):=\cos \left(2^{l} \arccos (x / 2)\right)$ defined on the interval $[-2,2]$. This follows from

$$
\begin{aligned}
\left\{x_{k}\right\}_{k=0}^{2^{l}} & =\{-2\} \cup\left\{2 \cos \pi c_{k}\right\}_{k=0}^{2^{l}-1}=\{-2\} \cup\left\{2 \cos \left(\pi 2^{-l} b(l, k)\right)\right\}_{k=0}^{2^{l}-1} \\
& =\{-2\} \cup\left\{2 \cos \frac{\pi k}{2^{l}}\right\}_{k=0}^{2^{l}-1}=\left\{2 \cos \frac{\pi k}{2^{l}}\right\}_{k=0}^{2^{l}} \cdot \square
\end{aligned}
$$

Example 1.4 suggests that the nodes $x_{k}$ can be thought of as being determined by a suitable enumeration of the Chebyshev points $\left\{2 \cos \left(\pi k / 2^{l}\right)\right\}_{k=0}^{2^{l}}$. 
In Section 2 we use well-known results on sets of equidistributed points to show that $q_{n-1}$ converges maximally to $f$ as $n$ increases. There, we also show that if the capacity $c$ of $\Omega$ is one, then $q_{n-1}$ is not very sensitive to perturbations of $f$.

Computed examples are presented in Section 3. The examples include comparisons with another enumeration of Fejér points, as well as comparisons of polynomials in Newton form with polynomials in Lagrange and barycentric forms. In Section 3 we also comment on the computation of Fejér points.

By the maximal convergence of $q_{n-1}$ to $f$ on $\Omega$ as $n$ increases, and by results on overconvergence of Walsh [12, Theorem 6, p. 78], it follows that the $q_{n-1}$ converge to an analytic continuation of $f$ in the exterior of $\Gamma$ as $n$ increases. Hence, the $q_{n-1}$ can be used to compute analytic continuations. A different method for analytic continuation based on summability theory has been described by Eiermann and Niethammer [3]. It would appear that the nodes defined by (1.10) and (1.11) would also be applicable in the method of [3].

2. Convergence and Stability. We formulate the convergence properties of the $q_{n-1}$ as a lemma.

LEMMA 2.1. Let $\Omega$ be a compact set bounded by a Jordan curve $\Gamma$, or let $\Omega=\Gamma:=[-2,2]$. Assume that $f$ is analytic on $\Omega$ and let $\rho(f)>1$ be the largest constant such that $f$ is analytic in the interior of the curve $\Gamma_{\rho(f)}$. Let $q_{n-1}$ be defined by (1.7), where the interpolation points (1.10) are used if $\Gamma$ is a Jordan curve, and the interpolation points (1.11) are used if $\Gamma=[-2,2]$. Then $q_{n-1}$ converges maximally to $f$, i.e.,

$$
\varlimsup_{n \rightarrow \infty}\left\|f-q_{n-1}\right\|_{\Gamma}^{1 / n}=1 / \rho(f) .
$$

Proof. The van der Corput sequence $\left\{c_{k}\right\}_{k=0}^{\infty}$ is uniformly distributed, in the sense of Weyl, on the interval [0,1], see [10, Chapter 1], [12, Chapter 7.5]. Therefore, the nodes defined by (1.10) or (1.11) satisfy the conditions of [5, Satz 2, p. 67]. This shows maximal convergence.

The property that a sequence is uniformly distributed says nothing about how its first $n$ elements are distributed. The next lemma indicates that the points $\xi_{k}, 0 \leq k<n$, are spread fairly uniformly over $\Gamma$ also for small values of $n$. We therefore can expect $q_{n-1}$ to yield a good approximation of $f$ already for modest values of $n$.

LEMMA 2.2. Let $\alpha$ and $\beta$ be nonnegative integers with $\beta>\alpha$. Then the set $\left\{\xi_{k}\right\}_{k=\alpha}^{\beta-1}$, defined by (1.10), can be written as the union of not more than $\gamma$ pairwise disjoint sets of Fejér points, with

$$
\gamma \leq\left\lfloor 2 \log _{2}\left(\frac{1}{2}(\beta-\alpha)+1\right)\right\rfloor
$$

where $\lfloor s\rfloor$ denotes the integer part of $s$.

Proof. From [4, Theorem 2.1] it follows that the set $\left\{\exp \left(i \theta_{k}\right)\right\}_{k=\alpha}^{\beta-1}$ can be written as the union of not more than $\gamma$ pairwise disjoint sets of equidistant points on the unit circle, with $\gamma$ bounded by (2.1). The lemma now follows from the definition of Fejér points (1.2).

We turn to the sensitivity of $q_{n-1}$ to perturbations in $f$. We wish to bound the propagated error in the divided differences $(1.7 \mathrm{~b})$ due to perturbations in the 
function values $f\left(\xi_{k}\right)$. The divided difference (1.7b) can be written as, see Davis $[2$, p. 40],

$$
\left[\xi_{0}, \xi_{1}, \ldots, \xi_{j}\right] f=\sum_{k=0}^{j} \frac{f\left(\xi_{k}\right)}{\prod_{l=0 ; l \neq k}^{j}\left(\xi_{k}-\xi_{l}\right)} .
$$

A lower bound for the products in (2.2) is obtained in two steps. We first consider the case when $\Omega$ is the unit disk. Results for the disk are then generalized to sets $\Omega$ with a smooth boundary by applying a theorem of Curtiss [1].

LEMMA 2.3. Let $w_{k}:=\exp \left(i \theta_{k}\right), k=0,1,2, \ldots$, where the $\theta_{k}$ are defined by (1.9). Then, for any $n \geq 1$ we have

$$
\prod_{\substack{j=0 \\ j \neq k}}^{n-1}\left|w_{k}-w_{j}\right| \geq n^{-1}, \quad 0 \leq k<n
$$

Proof. Let $l \geq 0$ be an integer such that $n \leq 2^{l}<2 n$. Then

$$
\prod_{\substack{j=0 \\ j \neq k}}^{n-1}\left|w_{k}-w_{j}\right|=\frac{\prod_{j=0 ; j \neq k}^{2^{l}-1}\left|w_{k}-w_{j}\right|}{\prod_{j=n}^{2^{l}-1}\left|w_{k}-w_{j}\right|}=\frac{2^{l}}{\prod_{j=n}^{2^{l}-1}\left|w_{k}-w_{j}\right|}
$$

We obtain by Lemma 2.2 that the set $\left\{w_{j}\right\}_{j=n}^{2^{l}-1}$ can be subdivided into $\gamma \leq$ $\left\lfloor 2 \log _{2}\left(\frac{1}{2}\left(2^{l}-n\right)+1\right)\right\rfloor$ pairwise disjoint subsets $\left\{w_{j, m}\right\}_{j=0}^{s_{m}-1}, 1 \leq m \leq \gamma$, of equidistant points. Hence,

$$
\begin{aligned}
\prod_{j=n}^{2^{l}-1}\left|w_{k}-w_{j}\right| & =\prod_{m=1}^{\gamma}\left(\prod_{j=0}^{s_{m}-1}\left|w_{k}-w_{j, m}\right|\right) \\
& =\prod_{m=1}^{\gamma}\left(\prod_{j=0}^{s_{m}-1}\left|w_{k} w_{0, m}^{-1}-w_{j, m} w_{0, m}^{-1}\right|\right) \\
& =\prod_{m=1}^{\gamma}\left|\left(w_{k} w_{0, m}^{-1}\right)^{s_{m}}-1\right| \leq 2^{\gamma}
\end{aligned}
$$

where we have used that $\left\{w_{j, m} w_{0, m}^{-1}\right\}_{j=0}^{s_{m}-1}$ are roots of unity. Therefore,

$$
\prod_{j=n}^{2^{l}-1}\left|w_{k}-w_{j}\right| \leq\left(\frac{1}{2}\left(2^{l}-n\right)+1\right)^{2} \leq \frac{1}{4}(n+1)^{2}
$$

and substitution into (2.3) yields

$$
\prod_{\substack{j=0 \\ j \neq k}}^{n-1}\left|w_{k}-w_{j}\right| \geq \frac{2^{l}}{\frac{1}{4}(n+1)^{2}} \geq \frac{4 n}{(n+1)^{2}} \geq n^{-1},
$$

for $0 \leq k<n$.

LEMMA 2.4 (Curtiss [1, Theorem 2]). Let $\Gamma$ be such that the derivative $\varphi^{\prime}(w)$ of the conformal mapping (1.1) is nonvanishing and of bounded variation for $|w|=1$. 
Then

$$
\lim _{n \rightarrow \infty} \frac{\prod_{j=0}^{n-1}(\varphi(w)-\varphi(\exp (2 \pi i j / n)))}{c^{n}\left(w^{n}-1\right)}=1,
$$

uniformly for $|w| \geq 1$, where $c$ denotes the capacity of $\Omega$.

LEMMA 2.5. Let $\Gamma$ be such that $\varphi^{\prime}(w)$ is nonvanishing and of bounded variation for $|w|=1$. Let the capacity of $\Omega$ be one. Then there are positive constants $\beta$ and $\delta$, independent of $n$ and $k$, such that

$$
\prod_{\substack{j=0 \\ j \neq k}}^{n-1}\left|\xi_{k}-\xi_{j}\right| \geq \beta n^{-\delta}
$$

for $0 \leq k<n$, where the $\xi_{k}$ are defined by (1.8)-(1.10).

Proof. Let $w_{k}:=\exp \left(i \theta_{k}\right), 0 \leq k<n$, where $\theta_{k}$ is defined by (1.9). Then $\xi_{k}=\varphi\left(w_{k}\right)$. Let $l \geq 0$ be an integer such that $n \leq 2^{l}<2 n$. Then

$$
\prod_{\substack{j=0 \\ j \neq k}}^{n-1}\left|\xi_{k}-\xi_{j}\right|=\prod_{\substack{j=0 \\ j \neq k}}^{n-1}\left|\varphi\left(w_{k}\right)-\varphi\left(w_{j}\right)\right|=\frac{\prod_{j=0 ; j \neq k}^{2^{l}-1}\left|\varphi\left(w_{k}\right)-\varphi\left(w_{j}\right)\right|}{\prod_{j=n}^{2^{l}-1}\left|\varphi\left(w_{k}\right)-\varphi\left(w_{j}\right)\right|}
$$

We use the notation of the proof of Lemma 2.3 and obtain by Lemma 2.2 , similarly as in the proof of Lemma 2.3 ,

$$
\prod_{j=n}^{2^{l}-1}\left|w_{k}-w_{j}\right|=\prod_{m=1}^{\gamma}\left(\prod_{j=0}^{s_{m}-1}\left|w_{k}-w_{j, m}\right|\right)
$$

where the sets $\left\{w_{j, m}\right\}_{j=0}^{s_{m}-1} \subset\left\{w_{j}\right\}_{j=n}^{2^{l}-1}, 1 \leq m \leq \gamma$, are disjoint sets of equidistant points on the unit circle, and $\gamma \leq 2 \log _{2}\left(\frac{1}{2}\left(2^{l}-n\right)+1\right)$. Hence,

$$
\prod_{j=n}^{2^{l}-1}\left|\varphi\left(w_{k}\right)-\varphi\left(w_{j}\right)\right|=\prod_{m=1}^{\gamma}\left(\prod_{j=0}^{s_{m}-1}\left|\varphi\left(w_{k}\right)-\varphi\left(w_{j, m}\right)\right|\right),
$$

where $\left\{\varphi\left(w_{j, m}\right)\right\}_{j=0}^{s_{m}-1} \subset\left\{\varphi\left(w_{j}\right)\right\}_{j=n}^{2^{l}-1}, 1 \leq m \leq \gamma$, are disjoint sets of Fejér points. By Lemma 2.4 there are constants $k_{0}, 0<\delta_{0} \leq \delta_{1}<\infty$, independent of $k$, but where $\delta_{0}$ depends on $k_{0}$, such that, for $k \geq k_{0}$,

$$
\prod_{j=0}^{k-1}|\varphi(w)-\varphi(\exp (2 \pi i j / k+i \alpha))| \geq \delta_{0} \prod_{j=0}^{k-1}|w-\exp (2 \pi i j / k+i \alpha)|
$$

and, for $k \geq 1$,

$$
\prod_{j=0}^{k-1}|\varphi(w)-\varphi(\exp (2 \pi i j / k+i \alpha))|<\delta_{1} \prod_{j=0}^{k-1}|w-\exp (2 \pi i j / k+i \alpha)|
$$

uniformly for $|w|=1$. The real constant $\alpha$ in (2.7) and (2.8) is arbitrary but fixed. From (2.6) and (2.8) we obtain, for $0 \leq k<n$,

$$
\prod_{j=n}^{2^{l}-1}\left|\varphi\left(w_{k}\right)-\varphi\left(w_{j}\right)\right| \leq \delta_{1}^{\gamma} \prod_{m=1}^{\gamma}\left(\prod_{j=0}^{s_{m}-1}\left|w_{k}-w_{j, m}\right|\right) \leq\left(2 \delta_{1}\right)^{\gamma},
$$


where

$$
\gamma \leq 2 \log _{2}\left(\frac{1}{2}\left(2^{l}-n\right)+1\right) \leq 2 \log _{2}\left(\frac{1}{2} n+\frac{1}{2}\right) \leq 2 \log _{2} n .
$$

Hence, we have obtained an upper bound for the denominator of (2.4). We now derive a lower bound for the numerator. From the conditions on $\varphi$ it follows that there is a constant $\delta_{2}>0$ such that

$$
|\varphi(w)-\varphi(\tilde{w})| \leq \delta_{2}|w-\tilde{w}|
$$

for all $w, \tilde{w}$ on the unit circle. By (2.7) and (2.11) it follows that

$$
\prod_{\substack{j=0 \\ j \neq k}}^{2^{l}-1}\left|\varphi(w)-\varphi\left(w_{j}\right)\right| \geq \frac{\prod_{j=0}^{2^{l}-1}\left|\varphi(w)-\varphi\left(w_{j}\right)\right|}{\delta_{2}\left|w-w_{k}\right|} \geq \delta_{0} \delta_{2}^{-1} \prod_{\substack{j=0 \\ j \neq k}}^{2^{l}-1}\left|w-w_{j}\right|
$$

for all $|w|=1$ and $2^{l} \geq k_{0}$. In particular,

$$
\prod_{\substack{j=0 \\ j \neq k}}^{2^{l}-1}\left|\varphi\left(w_{k}\right)-\varphi\left(w_{j}\right)\right| \geq \delta_{0} \delta_{2}^{-1} \prod_{\substack{j=0 \\ j \neq k}}^{2^{l}-1}\left|w_{k}-w_{j}\right|=2^{l} \delta_{0} \delta_{2}^{-1} \geq n \delta_{0} \delta_{2}^{-1}
$$

Combining (2.4), (2.9), (2.10) and (2.12) yields, for $n \geq k_{0}$,

$$
\prod_{\substack{j=0 \\ j \neq k}}^{n-1}\left|\xi_{k}-\xi_{j}\right| \geq \frac{n \delta_{0} \delta_{2}^{-1}}{n^{2\left(1+\log _{2} \delta_{1}\right)}}=\frac{\delta_{0} \delta_{2}^{-1}}{n^{1+2 \log _{2} \delta_{1}}}, \quad 0 \leq k<n
$$

where we have assumed that $\delta_{1} \geq 1 / 2$. This shows the lemma.

Combining Lemma 2.5 with (2.2) shows that perturbations in the function values $f\left(\xi_{k}\right), 0 \leq k<n$, are amplified at most polynomially in the divided differences (1.7b).

We are now in a position to bound the rate of growth with $n$ of the condition number of the mapping from the function values $f\left(\xi_{k}\right)$ to $q_{n-1}$. Related investigations for other polynomial bases have been carried out by Gautschi, see [6] and references therein.

Let $\Pi_{n-1}$ denote the set of polynomials of degree less than $n$, and define the vectors

$$
\begin{aligned}
\mathbf{f} & :=\left(f\left(\xi_{0}\right), f\left(\xi_{1}\right), \ldots, f\left(\xi_{n-1}\right)\right)^{T}, \\
\mathbf{c} & :=\left(\left[\xi_{0}\right] f,\left[\xi_{0}, \xi_{1}\right] f, \ldots,\left[\xi_{0}, \xi_{1}, \ldots, \xi_{n-1}\right] f\right)^{T},
\end{aligned}
$$

where we presently only assume that the $\xi_{j}$ are distinct points on $\Gamma$ and that the function $f$ is continuous on $\Gamma$. Introduce the mappings

$$
\begin{array}{ll}
T_{1}: \mathbb{C}^{n} \rightarrow \mathbb{C}^{n}, & T_{1} \mathbf{f}:=\mathbf{c} ; \\
T_{2}: \mathbb{C}^{n} \rightarrow \Pi_{n-1}, & \left(T_{2} \mathbf{c}\right)(z):=q_{n-1}(z) ; \\
T: \mathbb{C}^{n} \rightarrow \Pi_{n-1}, & T:=T_{2} \circ T_{1} .
\end{array}
$$

Equip the domains of $T_{1}, T_{2}$ and $T$, and the range of $T_{1}$, with the norm

$$
\|\mathbf{v}\|:=\max _{0 \leq j<n}\left|v_{j}\right|, \quad \mathbf{v}=\left(v_{0}, v_{1}, \ldots, v_{n-1}\right)^{T} \in \mathbb{C}^{n}
$$


and let the range of $T_{2}$ and $T$ have the norm (1.5). Let $T^{-1}$ denote the inverse of $T$, and let the norms $\left\|T_{1}\right\|,\left\|T_{2}\right\|,\|T\|$ and $\left\|T^{-1}\right\|$ be the induced operator norms. The condition number of $T$ is defined by

$$
\operatorname{cond}(T):=\|T\|\left\|T^{-1}\right\| \text {. }
$$

THEOREM 2.6. Let $\Gamma$ be such that $\varphi^{\prime}(w)$ is nonvanishing and of bounded variation on $|w|=1$. Assume that $\Omega$ is scaled to have capacity one, and let the interpolation points $\left\{\xi_{k}\right\}_{k=0}^{n-1}$ be given by (1.10). Then

$$
\lim _{n \rightarrow \infty} \operatorname{cond}(T)^{1 / n}=1 \text {. }
$$

Proof. We first bound $\left\|T_{1}\right\|^{1 / n}$ and $\left\|T_{2}\right\|^{1 / n}$. This yields the bound $\|T\|^{1 / n} \leq$ $\left\|T_{1}\right\|^{1 / n}\left\|T_{2}\right\|^{1 / n}$. By (2.2) we obtain

$$
\begin{aligned}
\left\|T_{1}\right\|^{1 / n} & =\max _{\|\mathbf{f}\|=1} \max _{0 \leq j<n}\left|\sum_{k=0}^{j} f\left(\xi_{k}\right) \prod_{\substack{l=0 \\
l \neq k}}^{j}\left(\xi_{k}-\xi_{l}\right)^{-1}\right|^{1 / n} \\
& \leq\left(n \max _{\substack{0 \leq k \leq j \\
0 \leq j<n}} \prod_{\substack{l=0 \\
l \neq k}}^{j}\left|\xi_{k}-\xi_{l}\right|^{-1}\right)^{1 / n}
\end{aligned}
$$

and Lemma 2.5 now yields

$$
\varlimsup_{n \rightarrow \infty}\left\|T_{1}\right\|^{1 / n} \leq 1
$$

Further,

$$
\left\|T_{2}\right\|^{1 / n}=\max _{\|\mathbf{c}\|=1}\left\|\sum_{j=0}^{n-1} c_{j} \prod_{k=0}^{j-1}\left(z-\xi_{k}\right)\right\|_{\Gamma}^{1 / n} \leq n^{1 / n} \max _{0 \leq j<n}\left\|\prod_{k=0}^{j-1}\left(z-\xi_{k}\right)\right\|_{\Gamma}^{1 / n} .
$$

Similarly as in the proof of Lemma 2.1, we obtain from [5, Chapter 2] that

$$
\varlimsup_{n \rightarrow \infty}\left\|\prod_{k=0}^{n-1}\left(z-\xi_{k}\right)\right\|_{\Gamma}^{1 / n}=1
$$

where we have used that the capacity of $\Omega$ is one. Hence,

$$
\varlimsup_{n \rightarrow \infty}\left\|T_{2}\right\|^{1 / n} \leq 1 \text {. }
$$

We turn to a bound for $T^{-1}$, and note that since $q_{n-1}\left(\xi_{j}\right)=f\left(\xi_{j}\right), 0 \leq j<n$, it follows that $\left\|q_{n-1}\right\|_{\Gamma} \geq\|\mathbf{f}\|$. Therefore,

$$
\left\|T^{-1}\right\|=\max _{\left\|q_{n-1}\right\|_{r}=1}\|\mathbf{f}\| \leq 1 .
$$

From (2.13)-(2.15) it follows that

$$
\varlimsup_{n \rightarrow \infty} \operatorname{cond}(T)^{1 / n} \leq 1 \text {. }
$$

The theorem now follows from (2.16) and from $\operatorname{cond}(T)=\|T\|\left\|T^{-1}\right\| \geq 1$.

We note that Theorem 2.6 can be shown independent of the scaling of $\Gamma$, but if $\Gamma$ is scaled to have capacity different from one, then the proof has to be modified. In particular, $\left\|T_{1}\right\|$ and $\left\|T_{2}\right\|$ will increase or decrease exponentially with $n$, and this can give rise to numerical difficulties, such as overflow or large propagated errors due to underflow. In the numerical examples of the next section we therefore have scaled $\Omega$ to have capacity one. 
3. Numerical Examples. In order to demonstrate that our interpolation scheme is quite insensitive to perturbations, we have carried out all computations in single-precision arithmetic, i.e., with only six significant decimal digits, on an IBM 3090 computer.

In all examples the approximation error is measured on a discrete point set on $\Gamma$. Let $g \in C(\Gamma)$. In case $\Gamma$ is a Jordan curve we use the seminorm

$$
\|g\|_{d}:=\max _{0 \leq k<10^{3}}\left|g\left(\varphi\left(\exp \left(2 \pi i k / 10^{3}\right)\right)\right)\right|
$$

and for $\Gamma=[-2,2]$ we use

$$
\|g\|_{d}:=\max _{0 \leq k \leq 10^{3}}\left|g\left(2 \cos \left(\pi k / 10^{3}\right)\right)\right| .
$$

In none of the examples does an increase in the number of points in the seminorms lead to different figures.

Example 3.1. In this example we compute the interpolation polynomial $q_{n-1}$ in Newton form defined by (1.7) for increasing values of $n$ and for four different choices of interpolation points. We approximate the function $f(z):=(z-1)^{-1}$ on the set $\Omega$ bounded by $\Gamma:=\left\{w-\frac{1}{8} w^{-3}:|w|=1\right\}$, see Figure 3.1. Here $\varphi(w)=w-\frac{1}{8} w^{-3}$.

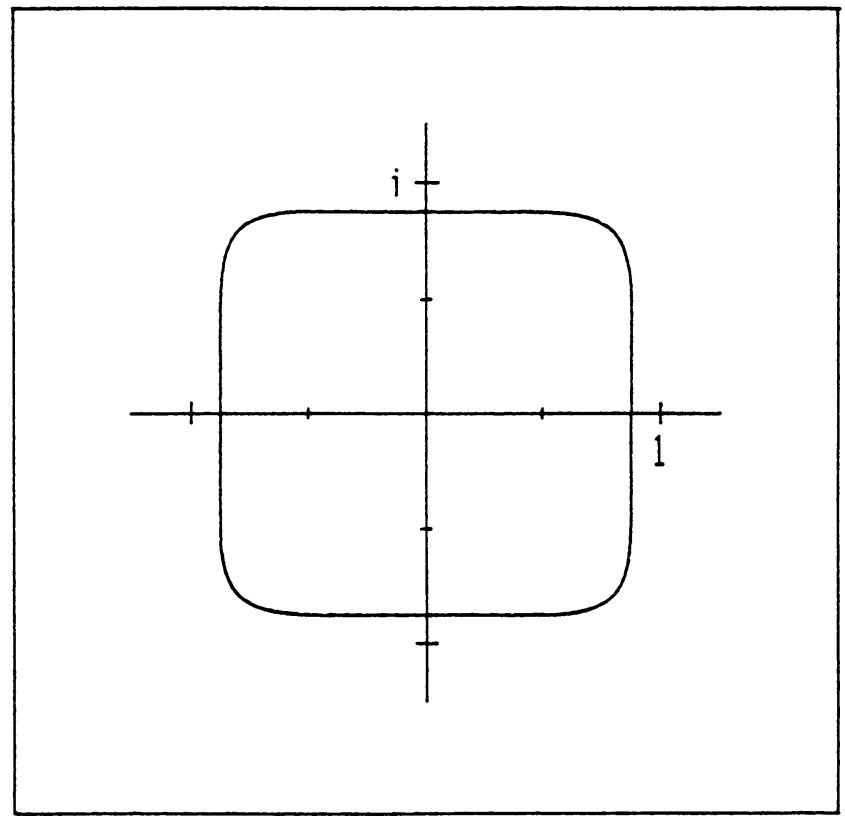

FIGURE 3.1

Boundary curve $\Gamma$.

The continuous curve of Figure 3.2 shows the error $\log _{10}\left\|f-q_{n-1}\right\|_{d}$ for $1 \leq$ $n \leq 128$, where the interpolation points $\xi_{k}$ defined by $(1.10)$ are used.

The $\xi_{k}$ are obtained by a particular enumeration of Fejér points. A possibly more natural enumeration defines the following interpolation points $\xi_{k}^{\prime}$. The enumeration is proposed in [3]. Introduce

$$
\left\{\begin{array}{l}
\theta_{0}^{\prime}:=0 \\
\theta_{k-1}^{\prime}:=2 \pi(2 l-1) / 2^{j+1}, \quad k>1,
\end{array}\right.
$$


where $j$ and $l$ are the unique integers such that $2^{j}<k \leq 2^{j+1}$ and $k=2^{j}+l, 1 \leq$ $l \leq 2^{j}$. Now let

$$
\xi_{k}^{\prime}:=\varphi\left(\exp \left(i \theta_{k}^{\prime}\right)\right), \quad k \geq 0 .
$$

The $\xi_{k}^{\prime}$ satisfy $\left\{\xi_{k}^{\prime}\right\}_{k=0}^{2^{m}-1}=\left\{\xi_{k}\right\}_{k=0}^{2^{m}-1}$ for all integers $m \geq 0$. However, it is easily seen that the $\theta_{k}^{\prime}$ are not uniformly distributed, in the sense of Weyl, on $[0,2 \pi]$. The dashed curve (on top) in Figure 3.2 shows $\log _{10}\left\|f-q_{n-1}\right\|_{d}$ where $q_{n-1}$ is obtained by interpolation in $\left\{\xi_{k}^{\prime}\right\}_{k=0}^{n-1}$. The curve shows that no convergence is achieved, and that the computations are sensitive to round-off errors. The dashed curve would in exact arithmetic touch the continuous one for $n=2^{m}, m=0,1,2, \ldots$, and in particular for $n=64$. The value of $\left\|f-q_{n-1}\right\|_{d}$ is very large for $n \geq 70$. For these $n$ values the dashed curve is not shown.

The dotted curve (on bottom) in Figure 3.2 shows $\log _{10}\left\|f-q_{n-1}\right\|_{d}$ where $q_{n-1}$ for every $n$ interpolates $f$ in a set of Fejér points $\left\{\xi_{j, n}\right\}_{j=0}^{n-1}$. Hence, for every $n$ all terms in (1.7) have to be recomputed. The points $\xi_{j, n}, 0 \leq j<n$, are obtained by renumbering $z_{j, n}, 0 \leq j<n$, defined by (1.2) with $\alpha=0$, using the bit reversal function $b(j, l)$ as follows. A renumbering of the $z_{j, n}$ is necessary for reasons of numerical stability, see below.

Algorithm 3.1 for renumbering Fejér points.

: definition of $\xi_{j, n}, 0 \leq j<n$ :

input $n \geq 1$, set of Fejér points $\left\{z_{j, n}\right\}_{j=0}^{n-1}$;

: let $l \geq 0$ be the unique positive integer such that $2^{l-1}<n \leq 2^{l}$ :

$k:=0$;

for $j:=0,1,2, \ldots, 2^{l}-1$ do

$$
\begin{aligned}
& \text { begin } \\
& \text { if } b(j, l)<n \text { then } \xi_{k, n}:=z_{b(j, l), n} ; k:=k+1 \text {; } \\
& \text { end }
\end{aligned}
$$

Figure 3.2 shows that the error obtained by interpolating in $\left\{\xi_{j, n}\right\}_{j=0}^{n-1}, n=$ $1,2,3, \ldots$, is not much smaller than when the nodes (1.10) are used. The computational effort, however, is much larger.

Finally, we determine the interpolation error when $q_{n-1}$ interpolates $f$ in $\left\{z_{j, n}\right\}_{j=0}^{n-1}, n=1,2,3, \ldots$ The corresponding error curve is not shown in Figure 3.2. The computational effort is comparable to using the nodes $\left\{\xi_{j, n}\right\}_{j=0}^{n-1}$, $n=1,2,3, \ldots$. However, the computations suffer from numerical instability. We obtain $\left\|f-q_{31}\right\|_{d}>1$ and $\left\|f-q_{63}\right\|_{d}>10^{8}$. The reason for this instability is that subsets $\left\{z_{j, n}\right\}_{j=0}^{k-1}, k=1,2, \ldots, n-1$, are unsuitably distributed on $\Gamma$. These latter computations motivated Algorithm 3.1.

The example shows that the nodes defined by (1.10) yield good accuracy and that the computations are quite insensitive to round-off errors.

Example 3.2. In this example we use nodes (1.10) and compare different representations of the interpolation polynomial: the Newton form (1.7), the Lagrange form

$$
l_{n-1}(z):=\sum_{k=0}^{n-1} f\left(\xi_{k}\right) \prod_{\substack{j=0 \\ j \neq k}}^{n-1} \frac{z-\xi_{j}}{\xi_{k}-\xi_{j}}
$$




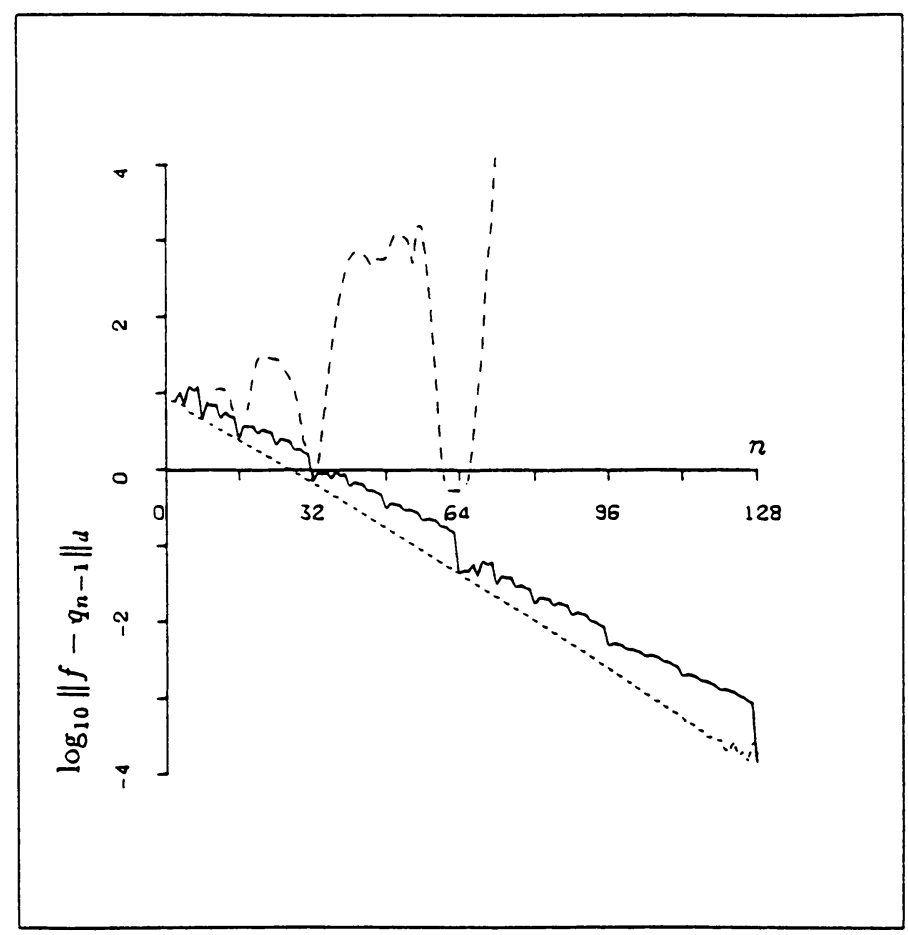

FIGURE 3.2

Newton polynomial $q_{n-1}$ defined by three choices of interpolation points.

and the barycentric form

$$
b_{n-1}(z):=\frac{\sum_{k=0}^{n-1} f\left(\xi_{k}\right)\left(z-\xi_{k}\right)^{-1} \prod_{j=0 ; j \neq k}^{n-1}\left(\xi_{k}-\xi_{j}\right)^{-1}}{\sum_{k=0}^{n-1}\left(z-\xi_{k}\right)^{-1} \prod_{j=0 ; j \neq k}^{n-1}\left(\xi_{k}-\xi_{j}\right)^{-1}} .
$$

The barycentric form is insensitive to perturbations, see Henrici [8, p. 237] and Werner [13]. However, due to perturbations, $b_{n-1}(z)$ may become rational, i.e., $b_{n-1}(z)$ may have poles in the finite plane. The computations with $b_{n-1}(z)$ can be arranged so that the computational effort required to form and evaluate $b_{n-1}(z)$ is of the same order of magnitude as for the Newton form [13].

Figure 3.3 shows the graphs of $\log _{10}\left\|f-q_{n-1}\right\|_{d}, \log _{10}\left\|f-l_{n-1}\right\|_{d}$ and $\log _{10}\left\|f-b_{n-1}\right\|_{d}$ with $f$ and $\Gamma$ the same as in Example 3.1. The curves coalesce, which indicates that the Newton form is as stable as the two other forms.

Example 3.3. In this example we approximate $f(x):=\left(1+2 x^{2}\right)^{-1}$ on the interval $\Omega=\Gamma:=[-2,2]$ by interpolation in the nodes (1.11). It follows from Example 1.1 that the capacity of $\Omega$ is one. We compare the Newton form $q_{n-1}$ with the Lagrange form $l_{n-1}$ and the barycentric form $b_{n-1}$. The latter forms are defined in Example 3.2. Figure 3.4 shows $\log _{10}\left\|f-q_{n-1}\right\|_{d}$ (continuous curve), $\log _{10}\left\|f-l_{n-1}\right\|_{d}$ (dashed curve) and $\log _{10}\left\|f-b_{n-1}\right\|_{d}$ (dotted curve). Again, the Newton form is seen to be quite insensitive to round-off errors. Indeed, we have evaluated $\log _{10}\left\|f-q_{n-1}\right\|_{d}$ for $n$ up to 256 without obtaining large propagated errors due to round-offs.

Example 3.4. The principal branch of $f(z)=z^{1 / 2}$ is approximated on $\Omega=$ $\{z:|z-1| \leq 1\}$ by $q_{n-1}$ defined by interpolation in the nodes (1.10). $f(z)$ is not 


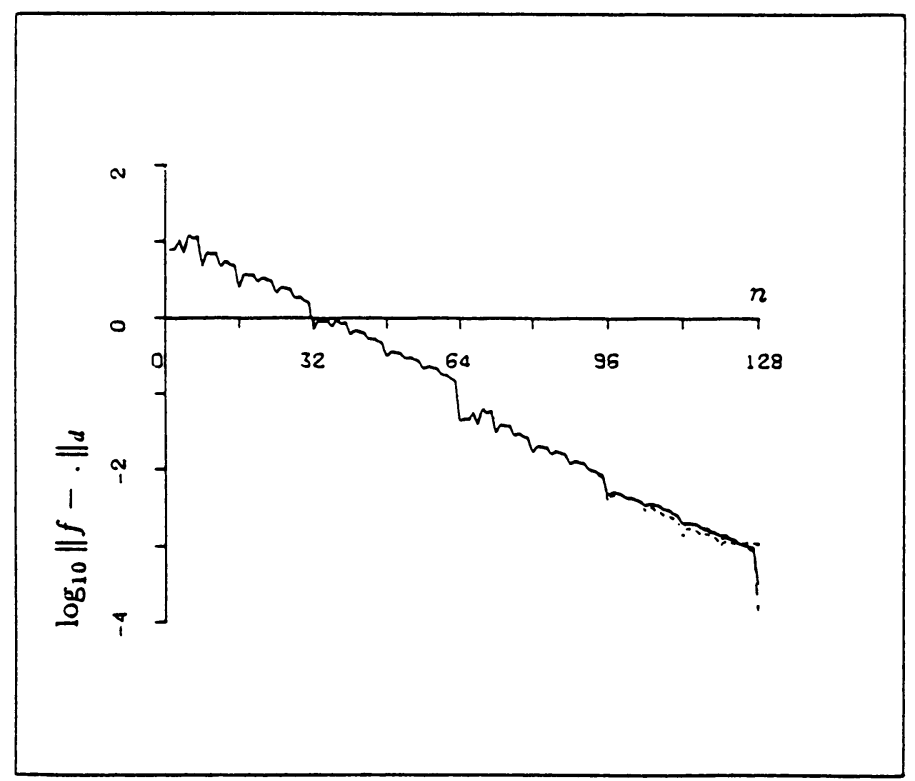

FIGURE 3.3

Newton, Lagrange and barycentric forms of the interpolation polynomial with nodes (1.10).

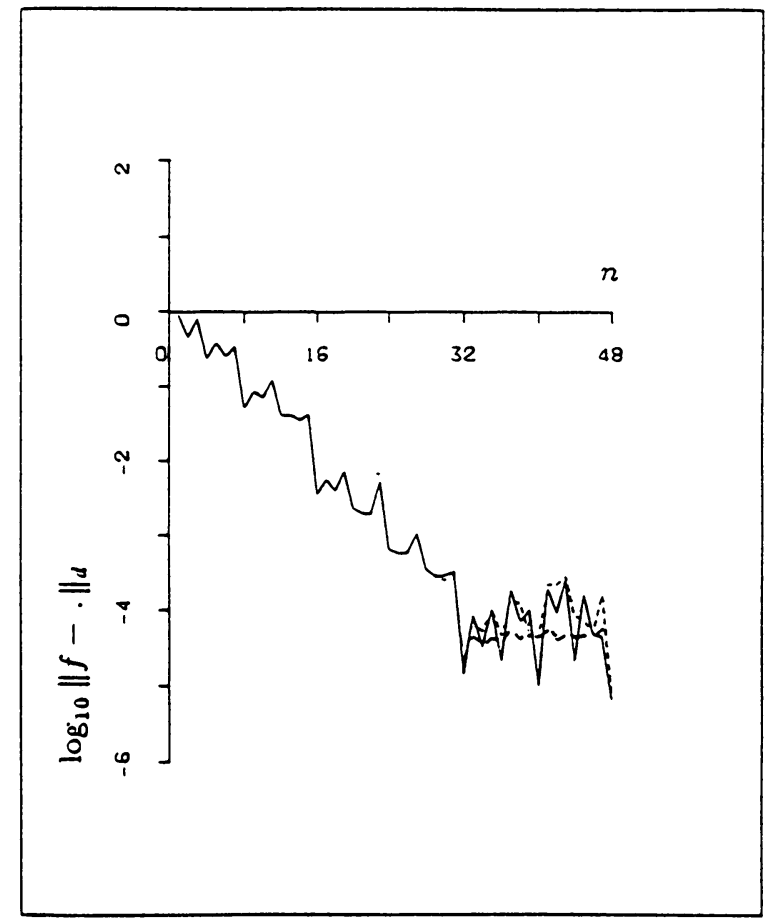

FIGURE 3.4

Newton, Lagrange and barycentric forms of the interpolation polynomial with nodes (1.11).

analytic on $\Omega$. A bound for $\left\|f-q_{n-1}\right\|_{d}$ when the interpolation points are equidistant is given by Geddes and Mason [7]. Figure 3.5 shows the error $\left\|f-q_{n-1}\right\|_{d}$. 
The error is seen to be smallest when the set of interpolation points $\left\{\xi_{j}\right\}_{j=0}^{n-1}$ can be written as the union of only few disjoint sets of equidistant nodes. The figure suggests that $q_{n-1}$ converges for a larger class of functions than considered in the present paper.

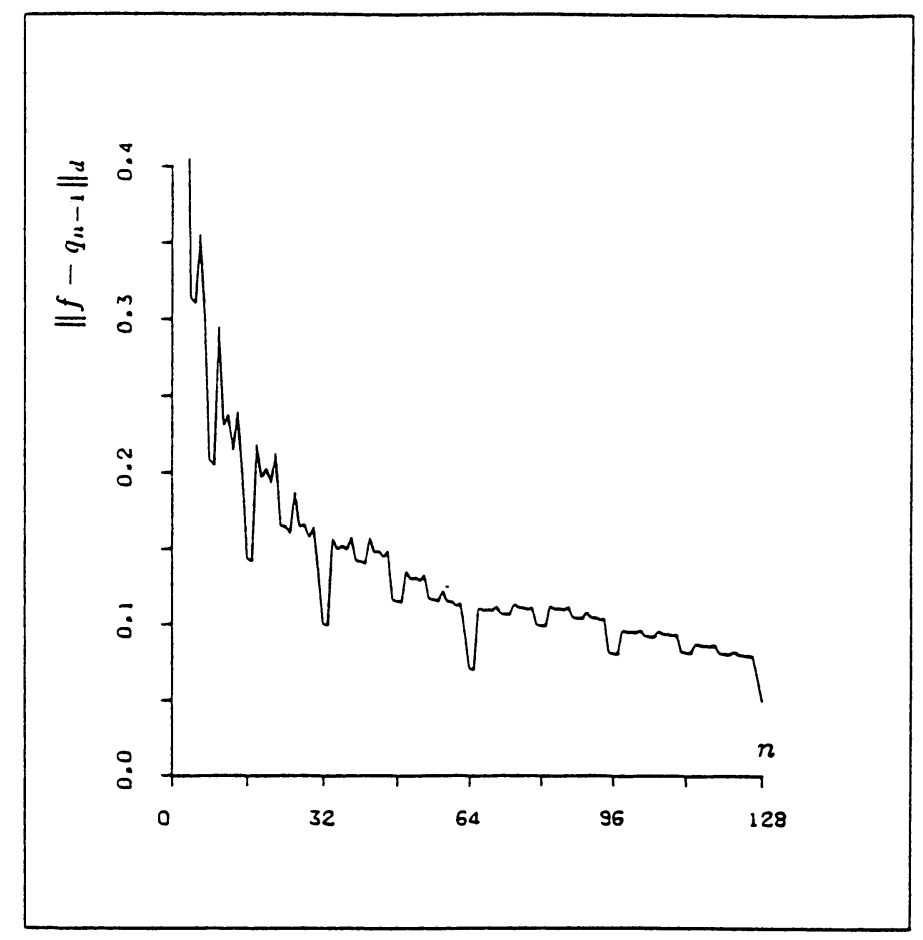

FIGURE 3.5

Approximation error for $f(z)=z^{1 / 2}$.

In our computed examples the conformal mappings $\varphi$ are explicitly known. This makes it simple to compute the nodes $\xi_{j}$. In general, the restriction of $\varphi$ to $\Gamma$ may have to be computed numerically. Recent surveys of numerical methods for this purpose are given by Henrici [9] and Trefethen [11].

4. Conclusion. Let $f$ be analytic on a compact simply connected set $\Omega$ in the complex plane. We consider the approximation of $f$ on $\Omega$ by interpolation polynomials $q_{n-1}$, where we describe a selection of interpolation points that allows us to represent $q_{n-1}$ in Newton form and to show maximal convergence. The interpolation points are suitably ordered points in certain sets of Fejér points. Maximal convergence is shown for sets $\Omega$ bounded by a Jordan curve. Numerical examples indicate that the scheme proposed yields accurate polynomial approximation when $\Omega$ is an interval, too. The propagation of errors in $f$ is also considered.

Acknowledgment. The authors would like to thank Professor Gaier for valuable comments. 
Bergen Scientific Centre

Allégaten 36

N-5007 Bergen, Norway

1. J. H. CURTISS, "Riemann sums and the fundamental polynomials of Lagrange interpolation," Duke Math. J., v. 8, 1941, pp. 525-532.

2. P. J. DAVIS, Interpolation and Approximation, Blaisdell, New York, 1963.

3. M. EIERMANN \& W. NIETHAMMER, "Interpolation methods for numerical analytic continuation," in Multivariate Approximation Theory II (W. Schempp and K. Zeller, eds.), ISNM 61, Birkhäuser, Basel, 1982.

4. B. FisChER \& L. REICHEL, "A stable Richardson iteration method for complex linear systems," Numer. Math., v. 54, 1988, pp. 225-242.

5. D. GAIER, Vorlesungen über Approximation im Komplexen, Birkhäuser, Basel, 1980.

6. W. GAUTSCHI, "Questions of numerical condition related to polynomials," in Studies in Numerical Analysis (G. H. Golub, ed.), Math. Assoc. Amer., 1984, pp. 140-177.

7. K. O. GEDDES \& J. C. MASON, "Polynomial approximation by projections on the unit circle," SIAM J. Numer. Anal., v. 12, 1975, pp. 111-120.

8. P. HENRICI, Essentials of Numerical Analysis, Wiley, New York, 1982.

9. P. Henrici, Applied and Computational Complex Analysis, vol. 3, Wiley, New York, 1986.

10. E. HLAWKa, The Theory of Uniform Distribution, Academic Publishers, Berkhamsted, 1984.

11. L. N. TREFethen (Ed.), "Numerical conformal mapping," Special Issue of J. Comput. Appl. Math., v. 16, 1986.

12. J. L. WALSH, Interpolation and Approximation by Rational Functions in the Complex Domain, 3rd ed., Amer. Math. Soc., Providence, RI, 1960.

13. W. Werner, "Polynomial interpolation: Lagrange versus Newton," Math. Comp., v. 43, 1984, pp. 205-217. 